\title{
BMJ Open Interface management concepts in healthcare for rare diseases in Germany: a study protocol for a mixed-methods study to develop best practice recommendations
}

\author{
Laura Inhestern (1) , David Zybarth, Ramona Otto, Maja Brandt, Martin Härter, \\ Corinna Bergelt
}

To cite: Inhestern L, Zybarth D, Otto R, et al. Interface management concepts in healthcare for rare diseases in Germany: a study protocol for a mixed-methods study to develop best practice recommendations. BMJ Open 2020;10:e040470. doi:10.1136/ bmjopen-2020-040470

- Prepublication history for this paper is available online. To view these files, please visit the journal online (http://dx.doi. org/10.1136/bmjopen-2020040470).

Received 14 May 2020

Revised 07 September 2020

Accepted 28 October 2020
Check for updates

(C) Author(s) (or their employer(s)) 2020. Re-use permitted under CC BY-NC. No commercial re-use. See rights and permissions. Published by BMJ.

Department of Medical Psychology, University Medical Center Hamburg-Eppendorf, Hamburg, Germany

Correspondence to

Laura Inhestern;

l.inhestern@uke.de

\section{ABSTRACT}

Introduction Patients and families affected by a rare disease are burdened in multiple ways. Functional interface management can unburden patients or relatives from the need to be solely accountable for the navigation through the healthcare system. This study aims at (1) providing an assessment of approaches and interface management concepts in the care of rare diseases, (2) evaluating selected existing approaches and concepts and (3) developing best practice recommendations for interface management.

Methods and analysis We will conduct a mixed-methods study with three phases. In phase 1, we will develop a tool to assess existing concepts of interface management for rare diseases based on a literature search and an expert workshop. The tool will be applied in a telephone survey with representatives of centres or clinics of expertise for rare diseases (target: $n=100$ ) and cooperating practitioners (target: $n=60$ ). Based on the results of phase 1, we will select four to six centres of expertise with interface management concepts, which will be evaluated extensively in phase 2 . For the evaluation, we will conduct semistructured interviews with practitioners cooperating with centres or clinics for rare diseases (target: $n=50$ ), a paper-based survey including patients or parents/legal guardians (target: $n=300$ ) from the selected centres or clinics, and semistructured interviews with patients or parents/legal guardians (target: $n=50$ ). The final phase of the study will be an integration of results from phases 1 and 2 to develop best practice recommendations for interface management in healthcare of rare diseases. In a concluding expert workshop, recommendations will be presented and finalised.

Ethics and dissemination This study was approved by the Local Psychological Ethics Committee of the Center for Psychosocial Medicine of the University Medical Center Hamburg-Eppendorf (LPEK-0062). The findings of our study will be presented on national and international conferences and published in scientific, peer-reviewed journals. To assure that centres for rare diseases get access to the study results, centres are invited to send a representative to a final expert workshop in phase 3 . Moreover, an executive summary will be provided and sent to relevant stakeholders.
Strengths and limitations of this study

- Based on three study phases, including qualitative and quantitative methods as well as the perspective of representatives of centres or clinics for rare diseases, practitioners and patients a rigorous development of best practice recommendations for interface management is assured.

- The results will allow healthcare providers to improve their interface management and, hence, to improve the journey through the healthcare system for patients with rare diseases and their relatives.

- Since we aim at including all centres for rare diseases in Germany, we will depend on their commitment for participation, and we may have a selection bias, for example, due to social desirability of study participants.

Trial registration number German Clinical Trials Registry (DRKS00020488).

\section{INTRODUCTION}

According to the European definition, a disease is defined as rare if $\leq 5$ in 10000 people are diagnosed with that disease. ${ }^{1}$ Currently, about 30 million people in the European Union (EU) ${ }^{1}$ and about 2.4-5 million people in Germany ${ }^{2}$ are affected by one of about 6000 to 8000 known rare diseases. ${ }^{1}$

Besides physical and mental constraints, living with a rare disease can be associated with social consequences such as stigma or financial drawbacks. ${ }^{34}$ Moreover, individuals with rare diseases can experience difficulties in their healthcare. ${ }^{5}$ Due to lack of knowledge (eg, in general practitioners (GPs) and the general population) and inefficient diagnostic ways, it can take several years for patients to receive accurate diagnosis. ${ }^{67}$ After diagnosis, receiving healthcare might be 
Table 1 Description of centres for rare diseases ${ }^{13}$

\begin{tabular}{lll}
\hline Patient group & Structure & Tasks \\
\hline Type A centre & & \\
$\begin{array}{l}\text { Patients with unclear diagnosis or an } \\
\text { undiagnosed disease }\end{array}$ & Comprises at least three B centres & Non-disease specific structure (eg, \\
& & $\begin{array}{l}\text { coordinator, interdisciplinary case } \\
\text { conferences) }\end{array}$ \\
& & Provision of education and teaching for \\
undergraduate medical students & Clinical research and basic research \\
& & acitivities
\end{tabular}

\section{Type B centre}

Patients diagnosed with a rare disease $\quad$ Integration into hospital setting $\quad$ Provision of inpatient and outpatient
or a clear suspected diagnosis multidisciplinary healthcare

Type C centre
$\begin{aligned} & \text { Patients diagnosed with a rare disease } \\ & \text { or a clear suspected diagnosis allowing } \\ & \text { access to healthcare near the patient's } \\ & \text { residence }\end{aligned}$$\quad \begin{aligned} & \text { Specialised clinics or specialised } \\ & \text { practitioners }\end{aligned} \quad \begin{aligned} & \text { Provision of outpatient care located near } \\ & \text { the patient's residence }\end{aligned}$

difficult in the home area of the patients, and patients often need to travel far to get access to appropriate treatment and care. ${ }^{89}$ Patients with rare diseases report diverse healthcare needs, for example, information on care facilities or psychological counselling. ${ }^{10}$ Recent studies show that more difficulties regarding a smooth flow through the healthcare system are associated with a reduced quality of life. $^{11}$

Due to the high burden of these patients, in 2009 the EU released several recommendations for its member states to improve the situation of individuals with rare diseases, and national action plans have been announced. ${ }^{12}$ The German National Plan of Action for People with Rare Diseases was published in 2013 and comprises 52 proposed actions, ${ }^{13}$ including a model of care delivery based on centres for rare diseases to structure and aggregate competencies. The national plan of action recommends centres for rare diseases on three levels based on the spectrum of their service (A centres, B centres and C centres) (table 1$).^{13}$

So far, no formal accreditation or certification body for centres of rare diseases has been implemented, but centres define themselves as A centres or B centres according to the catalogue of requirements from the national plan of action.

Currently, there are about 32 centres for rare diseases registered in the se-atlas, a platform for mapping healthcare providers to individuals with rare diseases in Germany. ${ }^{14}$ Most of these centres comprise more than three B centres. However, not all are designated as A centres.

In the healthcare of rare diseases, generic integrated care models to structure the paths of the patients through the healthcare system are missing, and follow-up care outside the centres for rare diseases can be insufficient. At the same time, integrated care is particularly important in rare diseases due to the delay in diagnosis and, if diagnosed, highly specialised demands regarding treatment and monitoring. ${ }^{15}$ In a sophisticated and highly specialised healthcare system, quality of care depends on the management of interfaces and may be impaired by deficits in communication and information transfer. ${ }^{16}$ Interfaces in healthcare of rare diseases are, for example, centre for rare diseases/primary healthcare provider, centre for rare diseases/patient, or centre for rare diseases/specialised clinic. ${ }^{13}$ To manage these interfaces, intersectional communication and coordination are mandatory to enable continuity of healthcare and to alleviate patients' or relatives' burden of being accountable for navigating through the healthcare system. ${ }^{17} 18$ Approaches for interface management range from implementation of health information technologies, care coordinators and one-stop clinics to standard operating procedures. ${ }^{15}$ 19-21 However, integrated care models have only been established for single rare diseases. ${ }^{20}$ The field is rather scattered, and overall guidelines including the shared experiences of individuals with rare diseases concerning medical and psychosocial consequences and best practice recommendations are missing.

The national plan of action has recommended to initiate a survey among practitioners and centres for rare diseases to identify relevant aspects to ensure cooperation (proposed action 17). ${ }^{13}$

Corresponding to this recommendation, our study focuses on concepts for interface management particularly between centres for rare diseases and practitioners in healthcare in Germany. The overall aim of our study is the development of best practice recommendations for interface management in healthcare of rare diseases based on two steps: first, the systematic analysis of existing concepts for interface management, including the identification of strengths and limitations, and second, the evaluation of the acceptance and feasibility of applied concepts. Specific research questions are the following: 
- Which approaches and solutions exist in the literature and current healthcare for patients suffering from rare diseases regarding the interface management between primary and specialist care?

- How do representatives of centres for rare diseases, GPs, specialists, and affected patients or parents/legal guardians evaluate existing approaches and concepts regarding their acceptance, feasibility and benefit?

- Which improvements to minimise interface problems do representatives of centres for rare diseases and practitioners (GPs and specialists) as well as affected patients or parents/legal guardians suggest?

\section{METHODS AND ANALYSIS}

This study protocol is written according the Standardized Protocol Items: Recommendations for Interventional Trials (SPIRIT) guidelines and addresses applicable recommended items for clinical trial protocols. ${ }^{22}$

\section{Study setting}

The study will be conducted in the Department of Medical Psychology at the University Medical Center HamburgEppendorf in Germany. The study is conducted in collaboration with the German National Alliance for Chronic Rare Diseases (ACHSE e.V.), the Martin Zeitz Center for Rare Diseases (a centre for rare diseases at the University Medical Center Hamburg-Eppendorf) and the Department of Pediatrics at the University Medical Center Hamburg-Eppendorf.

\section{Study design}

The study will be conducted in three phases (figure 1): phase 1 comprises a comprehensive inventory of existing concepts regarding interface management in healthcare of rare diseases. This phase provides an insight into and overview of how healthcare and interfaces are currently managed in centres for rare diseases. In phase 2, a differentiated evaluation of selected existing best practice concepts of interface management will be conducted. Including the patients' and practitioners' perspectives will provide important information on how the interfaces are working according to their experiences. And finally, based on the first two steps, specific recommendations for the implementation of a best practice model of interface management will be developed.

The key element of the project is a multiperspective analysis of the existing interface management concepts in centres for rare diseases in Germany. The study applies a mixed-methods design that includes quantitative and qualitative data collection.

\section{Phase 1: Collation of existing concepts of interface management} for rare diseases

Regarding the collation of existing concepts of interface management for rare diseases, we focus on the interface from primary healthcare to centres for rare diseases and back to primary healthcare (eg, strategies or workflow to link between different centres and primary healthcare providers). We aim at including A centres and B centres for rare diseases. Paediatric and adult healthcare as well as patients with a diagnosed or an undiagnosed rare disease, respectively, will be separately analysed to identify possible differences. The duration of phase 1 will be approximately 12 months.

The analysis of existing concepts will be conducted using a stepwise approach.

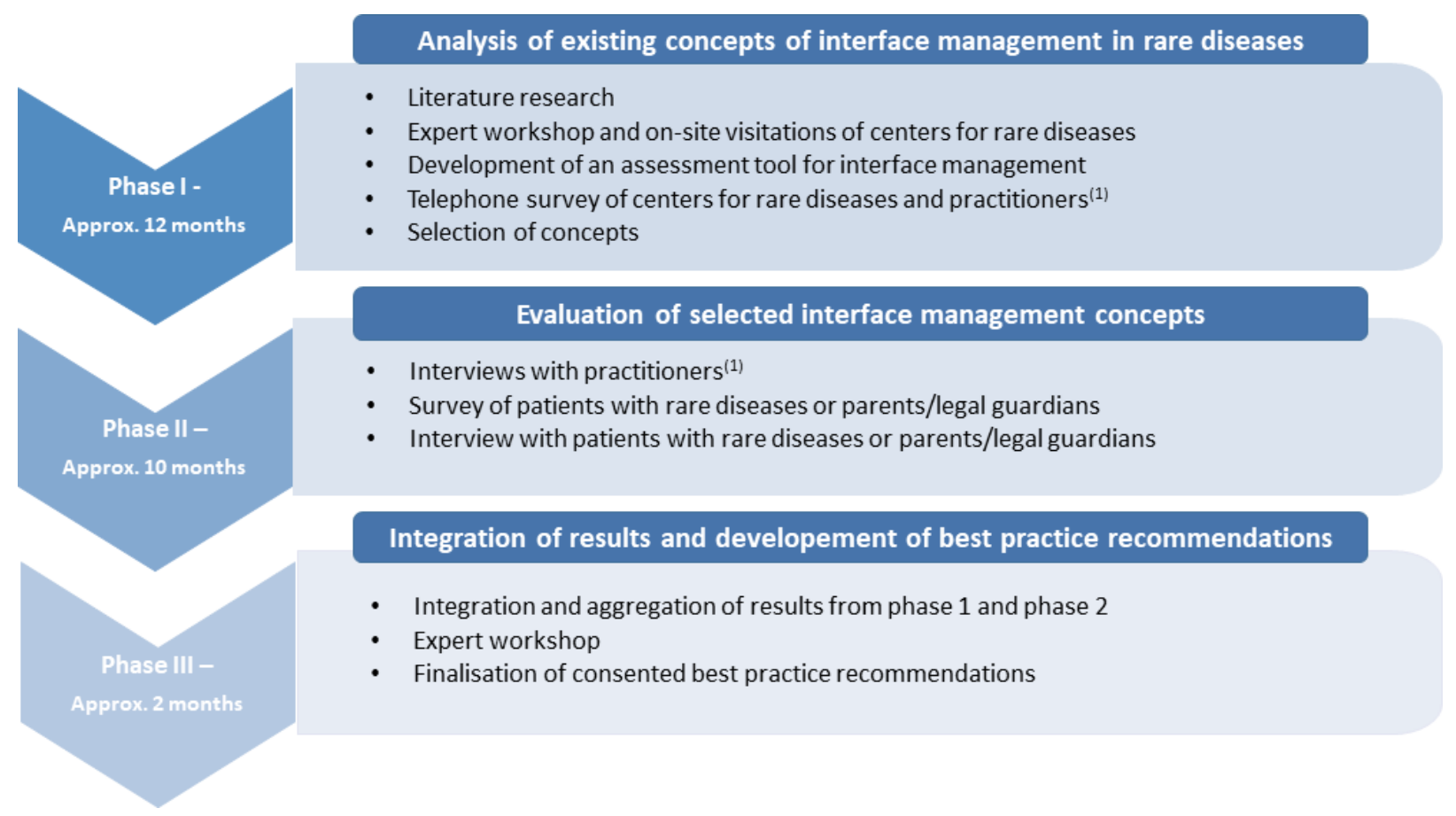

Figure 1 Flowchart of the study phases. (1) referring and cooperating primary and specialised practitioners. 
Step 1: Systematic search

1. A systematic search of the online presence of the centres for rare diseases in Germany will be conducted to identify instruments and questionnaires concerning interface management that are available on the websites of the centres. In addition, an online search concerning interface management tools for medical care in general or for chronic diseases will be performed.

2. A systematic search of international scientific publications concerning interface management in the medical care of rare diseases will be conducted (eg, PubMed and other databases) to identify and analyse concepts that may not have been implemented in Germany. The PubMed database will be searched using the search terms of interface management, care coordination, integrated care or intersectional communication, and rare disease, orphan disease or undiagnosed disease. No limitations regarding time will be adopted. The references of relevant literature will be searched for additional studies.

\section{Step 2: Expert workshop}

The centres for rare diseases will be invited to send one or two representatives for an expert workshop. The workshop will aim at exchanging experiences, gathering interface problems and providing possible solutions. To validly include the patient's perspective, the alliance for chronically rare diseases (ACHSE e.V.) will be asked to send three to four representatives as well. Furthermore, two to three representatives of the National Action League for People with Rare Diseases (NAMSE) and of the Federal Ministry of Health will be invited to participate.

\section{Step 3: On-site visitations}

Four to six centres for rare diseases will be visited by the project team to assess the implementation of interface management approaches. The visited centres should represent different regions of Germany. The method of on-site visitations is chosen based on the assumption that specific aspects and problems of interface management may not be adequately represented by written report or telephone surveys.

\section{Step 4: Development of an assessment tool for interface management}

Results of steps 1-3 (literature and online search, results of expert workshop and on-site visitations) will be collated by the study team, and relevant domains for interface management will be identified. Items covering the domains will be formulated by the study team, and a first draft of a structured assessment tool to evaluate the interface management for rare diseases will be developed. The assessment tool will be sent to the participants of the expert workshop and their feedback will be obtained. After adjustments based on the feedback, the assessment tool will be presented to three to five experts, discussed and finalised by expert consensus.
Step 5: Telephone survey of all centres of expertise for rare diseases and practitioners

All centres of expertise for rare diseases in Germany listed on a website collating all centres for rare diseases (se-atlas) will be invited for participation in a telephone survey using the developed and consented assessment tool. The telephone survey aims to investigate the interface management of the centres of expertise for rare diseases and to identify concepts, if applied in the centres.

In those centres that have a coordination site, the coordinator will be asked to participate and, additionally, to provide information on three to four centres of expertise for a specific rare disease or a disease group (B centres). In centres without the coordination site, three to four B centres will be randomly selected and surveyed.

Per centre of rare diseases, at least three participants should be surveyed. All in all, about 100 representatives of A centres and B centres should participate. Each participant will be asked to name the cooperating practitioners who also will be invited to participate in the survey. Overall, the final sample should comprise about 60 practitioners.

\section{Step 6: Selection of concepts}

Based on the scoring results from the telephone survey using the developed assessment tool (step 5), we will select four to six centres for rare diseases for further evaluation from the perspective of practitioners and individuals with rare diseases. Selection criteria will be based on the survey results on the description of interface management of the centres for rare diseases and on acceptance, feasibility and benefit of the concepts from the practitioners' perspective. Those centres with an established and working concept and structures to manage interfaces (eg, between sectors or between the centre and the patient) will be selected for study phase 2 .

Phase 2: Evaluation of selected centres regarding their interface management concepts

To evaluate four to six centres regarding their interface management concepts selected in phase 1 , we will include the perspective of patients with rare diseases or their parents/legal guardians, and the perspective of practitioners who refer patients and cooperate with selected centres. We will assess the experiences of interface management and collaboration with the centres for rare diseases and how it could be improved according to their needs from practitioners' and patients' perspectives. The duration of phase 2 will be approximately 10 months.

\section{Interviews with GPS or specialised practitioners}

The selected centres will be asked to name 10-15 cooperating GPs or specialised practitioners (target per centre: $\mathrm{n}=10$, total: $\mathrm{n}=50$ ). The identified practitioners will be invited to participate in a semistructured interview on their experiences with the interface concept of the respective centre for rare diseases. The interview guideline will address acceptance and benefits of interface 
concepts, the compatibility with medical practice and treatment processes, barriers and facilitators for the management of interfaces in medical care and suggestions for improvement.

\section{Survey of patients with rare diseases/relatives}

The selected centres will be asked to invite patients or parents/legal guardians of patients currently or formerly treated in the centre to participate in a cross-sectional survey (target per centre: $n=60$, total: $n=300$ ). The survey will comprise a questionnaire covering questions on the experiences of interface management as well as of existing and validated instruments on patient satisfaction, satisfaction with healthcare, psychosocial burden, quality of life and needs/unmet needs. In addition, relevant data regarding disease and healthcare history will be collected.

Survey participants will also be invited to participate in a semistructured interview (target per centre: $n=10$, total: $n=50$ interviews). The interviews will follow a guideline covering questions on the experiences of interface management and healthcare provision, barriers and facilitators for the management of interfaces in healthcare and suggestions for improvement.

\section{Data analysis}

All interviews will be conducted via telephone. The interviews will be recorded and transcribed verbatim and will be analysed by qualitative content analysis based on the approach of Mayring. ${ }^{23}$ The material will be coded using an inductive procedure. Categories obtained will be discussed by two separate researches to augment the validity and reliability of the coding guideline. Unclear category assignments are discussed until a consensus is reached. The analysis will be performed with the software program MaxQDA.

Quantitative data will be analysed using descriptive statistics to describe the study samples. Mean and SD will be reported for continuous data and frequencies and percentages for categorical data. Differences between patients and parents/legal guardians treated in different centres will be analysed using group comparisons $\left(\chi^{2}\right.$ test, $\mathrm{U}$ test or t-test depending on scale level). Outcomes will be patient satisfaction with healthcare and disease-related and healthcare-related burden. If applicable, diseaserelated parameters such as time until confirmed diagnosis or duration of hospital stays will also be included in the comparisons. To determine potential healthcare-related predictors of patients'/relatives' burden or patient satisfaction, multiple regression analyses will be applied. All statistical analyses will be performed using the software programme SPSS.

Qualitative and quantitative data will be synthesised after separate analyses of quantitative and qualitative data. The results on interface management will be related to each other and examined with regard to convergence, complementarity and discrepancy. Qualitative data may allow a further exploration of quantitative findings, increase understanding and support interpretation of results. ${ }^{24}$

Evaluation of not yet implemented interface management concepts If in step 6 of phase 1 an interface management concept is chosen, which has not been implemented until the time of our study, this concept will be evaluated using a qualitative approach based on expert interviews. We will invite experts from the field of healthcare provision for rare diseases (centres for rare diseases, NAMSE and ACHSE e.V.; target: $n=10$ ) to participate in an interview. The interview guideline will be developed according to the guideline for the primary and specialised healthcare providers in the evaluation of the selected interface management concepts.

Phase 3: Integration of results and development of best practice recommendations for interface management in healthcare of rare diseases

The final phase of the study will be based on the results of phases 1 and 2 and a concluding expert workshop. The duration of study phase 3 is approximately 2 months.

The results of study phase 1 (survey with representatives of the centres for rare diseases) and phase 2 (interviews with primary healthcare providers, survey and interviews with patients) will be integrated and aggregated. The findings will be aggregated by the study team and best practice recommendations will be drafted.

The recommendations will be presented to experts in the field of rare diseases (eg, patient representatives; representatives from the centres of expertise, ACHSE e.V. and NAMSE; Federal Ministry of Health) and discussed in an expert workshop. The aim of the workshop is to finalise and consent on explicit best practice recommendations for interface management in healthcare of rare diseases based on the considerations of the discussion.

\section{Data management and monitoring}

Members of the research team will continuously document data collection and manage the data collection at different phases.

Questionnaires will be entered in an SPSS database by research assistants. To assure high-quality data, double entry will take place for about $20 \%$ of the questionnaires and will be checked for mistakes. Data will only be accessible to members of the research team.

Adverse events will be monitored and documented, and the necessity of adaptation in the study process will be discussed within the research team.

\section{Patient and public involvement}

Patient organisations are systematically involved in the study. ACHSE e.V., the umbrella organisation of patient organisations, is a collaborator on the study. During study phases 1 and 3, representatives of the ACHSE e.V. will be invited to participate in the expert workshops. In phase 2 , patients' perspective is systematically included, and a crucial part of the evaluation of the interface management concepts. 


\section{ETHICS AND DISSEMINATION}

\section{Ethics approval and consent to participate}

The study has been approved by the Local Psychological Ethics Committee of the Center for Psychosocial Medicine of the University Medical Center Hamburg-Eppendorf (LPEK-0062). All participants will receive detailed study information. Informed consent will be obtained from study participants prior to participation in the study.

\section{Confidentiality}

Data protection is assured by pseudonymisation using a unique code. The code list can only be decrypted by members of the research team and will be destroyed after the end of data collection. Access to study data will be restricted by authorised access only for members of the research team.

\section{Dissemination}

The findings of our study will be presented on national and international conferences and published in peerreviewed scientific journals. To assure that centres for rare diseases get access to the study results, all centres are invited to send a representative to the final expert workshop in phase 3. Moreover, an executive summary will be provided and sent to all centres for rare diseases, all participating practitioners and NAMSE, as well as other relevant professional societies, for example, the German Society of Pediatrics and Adolescent Medicine. To make the results accessible and available to affected patients and families, an executive summary will be sent to ACHSE e.V. to distribute it to their member patient organisations.

\section{DISCUSSION}

The findings of our study will provide a systematic assessment of the current state of interface management in the context of rare diseases in Germany. A structured assessment tool to evaluate the interface management will be developed. The tool will focus on processes regarding the access to centres for rare diseases and information transfer to and from other healthcare providers. At the end of the study, consented best practice recommendations will allow healthcare institutions to improve their interface management and, hence, to improve the journey through the healthcare system for affected patients and their relatives.

\section{Strengths and limitations}

One strength of this study is the rigorous development of the assessment tool to measure interface management based on expert workshops with both representatives of centres for rare diseases and representatives of patient organisations. Important aspects from both perspectives will be represented in the tool.

Moreover, aiming at the inclusion of all centres for rare diseases in Germany, the study will provide a comprehensive assessment of interface management in centres for rare diseases in Germany. We will conduct an evaluation of the interface management in selected centres from the perspective of practitioners cooperating with the centres for rare diseases and patients or parents/legal guardians. This allows conclusions not only on the internal processes between healthcare providers but also on the impact on patients or parents/legal guardians themselves in their challenge to navigate through the healthcare system, which can be considered as another strength of the study.

One major limitation of the study is that the study success will depend on the commitment of participation of centres for rare diseases. It could be possible that we receive socially desirable answers, in particular from representatives of centres of rare diseases because they may know about best practice processes but do not include them in their daily routines. The study design aims at alleviating this possible bias by the inclusion of the perspective of referring primary care physicians and the perspective of the patient/parent/legal guardian in different study phases. However, we might also experience a selection bias in the recruitment or willingness of participation in practitioners cooperating with the centres for rare diseases and patients/relatives, for example, practitioners or patients with certain positive or explicitly negative experiences might be more likely to participate.

Another limitation is the method of expert workshops in phases 1 and 3. Participants will need to commit to participate, and there may be a limited reliability (eg, selection/ participation bias). At the same time, this method allows the inclusion of patient experts and healthcare experts in the development of recommendations for interface management.

Against the background of internationally different healthcare services, our findings may be relevant specifically for Germany. Still, difficulties in healthcare provision for patients with rare diseases are experienced across countries. ${ }^{25} 26$ Therefore, the results of our study and certain recommendations based on our findings may be relevant internationally.

Contributors $\mathrm{CB}$ and $\mathrm{MH}$ designed the study. $\mathrm{LI}, \mathrm{DZ}, \mathrm{MB}$ and $\mathrm{RO}$ were involved in the conception and design of the study. LI drafted the manuscript, which was modified and supplemented by all other authors. All authors were involved in the management and execution of the study. All authors were involved in revising the manuscript substantively, and read and approved the final manuscript.

Funding The study is funded by the Federal Ministry of Health (Bundesministerium für Gesundheit) in Germany. Representatives of our funding source will be invited to participate in the expert workshops and will be informed about the selection of interface management concepts for evaluation. However, the funding source is not involved in the study design, collection, analysis and interpretation of data, and writing the manuscript.

\section{Competing interests None declared.}

Patient and public involvement Patients and/or the public were involved in the design, or conduct, or reporting, or dissemination plans of this research. Refer to the 'Methods' section for further details.

Patient consent for publication Not required.

Provenance and peer review Not commissioned; externally peer reviewed.

Open access This is an open access article distributed in accordance with the Creative Commons Attribution Non Commercial (CC BY-NC 4.0) license, which permits others to distribute, remix, adapt, build upon this work non-commercially, and license their derivative works on different terms, provided the original work is 
properly cited, appropriate credit is given, any changes made indicated, and the use is non-commercial. See: http://creativecommons.org/licenses/by-nc/4.0/.

\section{ORCID iD}

Laura Inhestern http://orcid.org/0000-0003-2513-7954

\section{REFERENCES}

1 European Organization for Rare Diseases (EURODIS). What is a rare disease? Available: www.eurordis.org/about-rare-diseases [Accessed 30 Aug 2020].

2 Wetterauer B, Schuster R. Seltene Krankheiten. Bundesgesundheitsblatt Gesundheitsforschung Gesundheitsschutz 2008;51:519-28.

3 Pelentsov LJ, Fielder AL, Laws TA, et al. The supportive care needs of parents with a child with a rare disease: results of an online survey. BMC Fam Pract 2016;17:88.

4 von der Lippe C, Diesen PS, Feragen KB. Living with a rare disorder: a systematic review of the qualitative literature. Mol Genet Genomic Med 2017;5:758-73.

5 Schieppati A, Henter J-I, Daina E, et al. Why rare diseases are an important medical and social issue. The Lancet 2008;371:2039-41.

6 European Organization for Rare Diseases (EURODIS). The voice of 12.000 patients - experiences and expectations of rare disease patients on diagnosis and care in Europe. Paris; 2009.

7 Kohlschütter $A$, van den Bussche $\mathrm{H}$. [Early diagnosis of a rare disease in children through better communication between parents, physicians and academic centers]. Z Evid Fortbild Qual Gesundhwes 2019;141-142:18-23.

8 Reimer A, Bruckner-Tuderman L, Ott H. Mapping health care of rare diseases: the example of epidermolysis bullosa in Germany. Orphanet J Rare Dis 2018;13:197.

9 Liuccio M, Belotti R, Comune A, et al. Communication in rare diseases: a literature review. J Com Res 2015;7:215-23.

10 Babac A, Frank M, Pauer F, et al. Telephone health services in the field of rare diseases: a qualitative interview study examining the needs of patients, relatives, and health care professionals in Germany. BMC Health Serv Res 2018;18:99.

11 Bogart KR, Irvin VL. Health-Related quality of life among adults with diverse rare disorders. Orphanet J Rare Dis 2017;12:177.

12 Council of the European Union Council recommendation of 8 June 2009 on an action in the field of rare diesases (2009/C151/02). Official Journal of the European Union 2009.
13 . National action League for people with rare diseases, National plan of action for people with rare diseases. Bonn; 2013.

14 Haase J, Wagner TOF, Storf H. [se-atlas - the health service information platform for people with rare diseases : Supporting research on medical care institutions and support groups]. Bundesgesundheitsblatt Gesundheitsforschung Gesundheitsschutz 2017;60:503-9.

15 Breen C, Altman L, Ging J, et al. Significant reductions in tertiary hospital encounters and less travel for families after implementation of paediatric care coordination in Australia. BMC Health Serv Res 2018;18:751.

16 Kripalani S, LeFevre F, Phillips CO, et al. Deficits in communication and information transfer between hospital-based and primary care physicians: implications for patient safety and continuity of care. JAMA 2007;297:831-41.

17 Baumbusch J, Mayer S, Sloan-Yip I. Alone in a crowd? parents of children with rare diseases' experiences of Navigating the healthcare system. J Genet Couns 2018. doi:10.1007/s10897-018-0294-9. [Epub ahead of print: 21 Aug 2018]

18 Currie G, Szabo J. 'It is like a jungle gym, and everything is under construction': The parent's perspective of caring for a child with a rare disease. Child Care Health Dev 2019;45:96-103.

19 Kessel M, Hannemann-Weber H, Kratzer J. Innovative work behavior in healthcare: the benefit of operational guidelines in the treatment of rare diseases. Health Policy 2012;105:146-53.

20 Van Groenendael S, Giacovazzi L, Davison F, et al. High quality, patient centred and coordinated care for Alstrom syndrome: a model of care for an ultra-rare disease. Orphanet J Rare Dis 2015;10:149.

21 Grigull L, Mehmecke S, Rother A-K, et al. Common pre-diagnostic features in individuals with different rare diseases represent a key for diagnostic support with computerized pattern recognition? PLoS One 2019;14:e0222637.

22 Chan A-W, Tetzlaff JM, Altman DG, et al. SPIRIT 2013 statement: defining standard protocol items for clinical trials. Ann Intern Med 2013;158:200-7.

23 Mayring P. Qualitative content analysis: theoretical foundation basic procedures and software solution, 2014.

24 O'Cathain A, Murphy E, Nicholl J. Three techniques for integrating data in mixed methods studies. BMJ 2010;341:c4587.

25 Héon-Klin V. European reference networks for rare diseases: what is the conceptual framework? Orphanet J Rare Dis 2017;12:137.

26 Crowe AL, McKnight AJ, McAneney H. Communication needs for individuals with rare diseases within and around the healthcare system of Northern Ireland. Front. Public Health 2019;7:236. 